Bull. Austral. Math. Soc.

Vol. 40 (1989) [323-329]

\title{
GROWTH SEQUENCES OF FINITELY GENERATED GROUPS II
}

\author{
A.G.R. S'tewart and James Wiegold \\ For Bernhard Neumann with respect and affection
}

A study is made of the minimum number of generators of the $n$-th direct power of certain finitely generated groups.

\section{INTRODUCTION}

For a finitely generated group $G$, the growth sequence of $G$ is the sequence $\left\{d\left(G^{n}\right)\right\}$, where $d\left(G^{n}\right)$ denotes the minimum number of generators of the $n$-th direct power $G^{n}$ of $G$. A very tight description was given in [3-7] for the growth sequence of a finite group $G$; the main gross feature is that the sequence increases roughly logarithmically in $n$ if $G$ is perfect, while $d\left(G^{n}\right)=n d\left(G / G^{\prime}\right)$ for large enough $n$ if $G$ is not perfect. For infinite groups, the picture is less clear. It is again the case (see [8]) that $d\left(G^{n}\right)=n d\left(G / G^{\prime}\right)$ eventually if $G$ is not perfect, and that $\left\{d\left(G^{n}\right)\right\}$ is bounded above by a logarithmic function of $n$ if $G$ is perfect. The most important and apparently quite unattackable problem left is this: if $G$ has no finite images other that the identily, prove that $d\left(G^{n}\right)=d\left(G^{n+1}\right)$ for all large enough $n$.

The present article arose out of an unsuccessful attempt to answer a related problem.

Problem 1. Let $G$ be a finitely generated group having a non-trivial finite image. Is the growth sequence of $G$ eventually the sane as that of a finite image of $G$ ?

By the above renarks, this is true if $G$ is not perfect. The perfect case seems very hard, and we are far from a solution. Note that the positive solution to Problem 1 would entail the existence of a finite image whose growth sequence is eventually as fast as that of every finite image of $G$. This is true, at least in the case where $G$ has a unique non-lrivial inage of smallest order (which must, of course, be simple) - see Theorem A. Whether it is true in general involves the following sort of situation. Let $S$ and $T$ be 2-generator non-abelian finite simple groups of the same order $s$. The theorem in [0] shows that $d\left(S^{s^{n}}\right)=d\left(T^{n^{n}}\right)=n+2$ for all $n \geqq 0$, so that the growth sequences are

Received 6 April, 1989

We thank Dr. D. Meier for alerting us to some of the possible uses of Meier sets.

Cupyright Clearance Centre, Inc. Serial-fee code: $0004-9729 / 89 \$ \$ 2.00+0.00$. 
the same infinitely often. However, it could be that $d\left(S^{m}\right)>d\left(T^{m}\right)$ and $d\left(T^{n}\right)>d\left(S^{n}\right)$ for infinitely many values of $m$ and $n$; short of taking two different simple groups of the same order and examining Möbius functions (see[2]) of their subgroup lattices (a tall order!) we see no way of resolving this problem. Theorem A could be reformulated to take into account these possibilities, but we see no benefit in doing this.

A type of group where the finite image with fastest growth sequence is easily identified is the free square $G=B \star B$ of a non-abelian finite simple group $B$. It turns out to be $B^{2+a}$, where $a=\mid$ Aut $B \mid$ (Corollary to Theorem B). We believe the growth sequence of $G$ is eventually that of $B^{2+a}$. In any case, by [7],

$$
d\left(G^{n}\right) \geqq d\left(B^{(2+a) n}\right)>\log _{s} n+\log _{s}(2+a)+\log _{a} a
$$

where $s=|B|$. If it is true that $d\left(G^{n}\right)=d\left(B^{(2+a) n}\right)$ from some point on, then by [3, 4.4],

$$
d\left(G^{n}\right) \leqq \log _{8} n+\log _{8} a+\log _{8}(2+a)+1+\psi(n),
$$

where $\psi(n) \rightarrow 0$ as $n \rightarrow \infty$. In utmost generality, all that we can say is

$$
d\left(G^{n}\right) \leqq 2 d\left(B^{n}\right) \leqq 2\left(\log _{a} n+\log _{8} a+\psi(n)\right)
$$

Upper bounds for growth sequences can be attacked using a concept incorporated in the proof of Theorem 4.2 of [8], and discovered independently by David Meier (unpublished). In recognition of Dr. Meier's contribution we make the following definitions:

Definition 1: A subset $M$ of a group $G$ is said to be a Meier set for $G$ if

(i) for each non-trivial $x$ in $M,\langle x\rangle^{G}=G$,

(ii) for every pair of distinct elements $x, y$ of $M,\left\langle x y^{-1}\right\rangle^{G}=G$.

DEFINITION 2: The Meier number $\mu(G)$ is the largest cardinal of a Meier set of G.

Clearly, $G$ has Meier number 1 if and only if it is not the normal closure of a single element. Equally clearly, $\mu(B)=|B|$ for a simple group $B$.

The main general results using this concept are as follows.

THEOREM C. If $G$ is a finitely generated perfect group with $m=\mu(G)$ finite, then $d\left(G^{m n}\right) \leqq d\left(G^{n}\right)+1$ for all $n$. Thus $d\left(G^{n}\right)<d(G)+\log _{m} n+1$ for all $n$.

THEOREM D. If $G$ is a finite perfect group and $s$ the smallest order of a non-trivial image of $G$, then $\mu(G)=s$.

These two theorems combine to give elementary proofs of results in [5]. 
To improve on (1.1), it would suffice to prove that $B \star B$ has a Meier set with more than $\sqrt{|B|}$ elements. We believe this to be true, but even the verification for $A_{5}$ took a computer! Using a computer program devised by Dr. C.H. Houghton of University College Cardiff and implemented on the University of Zimbabwe computer with the help of Dr. P.J. Fish, Dr. J.A. Howarth and Mr. P.J. McBurney, a Meier set of 15 elements of length 2 has been found in $A_{5} \star A_{5}$. We thank all the four people mentioned for their invaluable assistance. For $G=A_{5} \star A_{5}$, we deduce from Theorem C that

$$
d\left(G^{n}\right)<5+\log _{15} n=5+\log _{15} 60 \log _{60} n
$$

for all $n$. This is better than (1.1); the dominant coefficient 2 has been reduced to $\log _{15} 60=1.5119 \ldots$, about half way to the 1 we would like it to be. The computer has also been used to check that $A_{5} \star A_{5}$ has no Meier set of order 60 consisting of elements of length 2. Meier sets with elements of higher lengths have not yielded to attack. In this context we ask:

Problem 2. For a finite non-abelian simple group $B$, is $\mu(B \star B)<|B|$ ? Note that every finite image of $B \star B$ has Meier number $|B|$, by Theorem $\mathrm{D}$.

It is clear that $\mu(B \star B)$ is at least as great as the cardinal of the smallest nontrivial Syluw subgroup of $B$, and thus certainly (to put it at its very crudest) at least 3. We thank the referee for pointing out that $\mu(B \star B)$ is at least the cardinal of a second largest Sylow subgroup of $B$, and thus certainly (to put it at its very crudest), at least 4 . We repeat our belief that the answer to our final problem is yes:

Problem 3. For every finite non-abelian simple group $B$, is there a Meier set of $B \star B$ consisting of elements of length 2 and of cardinal more than $\sqrt{|B|}$ ?

In a subsequent article, we hope to turn to the study of the growth sequence of a free product $G=A \star B$, where $A$ and $B$ are finite simple groups with $|A|<|B|$. The growth sequence of $G$ should eventually be the same as that of $A$, and the following is evidence, albeit thin, in favour of this. If $|B|$ has a prime divisor larger than $|A|$, it is evident that $G$ has a Meier set of cardinal $|A|$, so that $d\left(G^{n}\right)<d(G)+\log _{|A|} n+1$ for all $n$, by Theorem $C$. This means that $\left\{d\left(G^{n}\right)\right\}$ and $\left\{d\left(A^{n}\right)\right\}$ differ by at most a bounded quantity.

\section{ProOFs}

Theorem A. Let $G$ be a finitely generated perfect group having non-trivial finite images, suppose that $G$ has a unique non-trivial image $S$ of smallest order, and let $S^{\lambda}$ be the highest power of $S$ that is an image of $G$. Then the growth sequence of $S^{\lambda}$ is event ually as large as that of every finite inage of $G$; that is to say, there is a constant 
$K$ depending only on $G$ such that $d\left(B^{n}\right) \leqq d\left(S^{\lambda n}\right)$ for every finite image $B$ of $G$ and every $n \geqq K$.

Proof: Let $B$ be a non-trivial finite image of $G$, let $S_{1}, S_{2}, \ldots, S_{r}$ be the simple images of $B$, and $S_{1}^{\lambda_{1}}, S_{2}^{\lambda_{2}}, \ldots, S_{r}^{\lambda_{r}}$ the highest powers of $S_{1}, S_{2}, \ldots, S_{r}$ that are images of $B$. (The existence of $\lambda, \lambda_{1}, \ldots, \lambda_{r}$ follows since the growth sequence of every non-trivial finite group is unbounded [3].) We deal first with the case where $S$ is not one of $S_{1}, S_{2}, \ldots, S_{r}$. Since $d(B) \leqq d(G)$, we get from [5] and [3] that

$$
\begin{aligned}
d\left(B^{n}\right) & <d(B)+1+\log _{t} n \leqq d(G)+1+\log _{t} n, \\
d\left(S^{\lambda n}\right) & >\log _{8}(\lambda n),
\end{aligned}
$$

where $t=\min \left(\left|S_{1}\right|,\left|S_{2}\right|, \ldots,\left|S_{r}\right|\right)$ and $s=|S|$. Thus $d\left(S^{\lambda n}\right) \geqq d\left(B^{n}\right)$ as soon as $\log _{s}(\lambda n) \geqq \log _{t} n+d(G)+1$; since $s<t$, this certainly holds if $\log _{\theta}(\lambda n) \geqq$ $\log _{s+1} n+d(G)+1$. Note that $\lambda, s, d(G)$ depend only on $G$ and not on $B$.

Now suppose that $S$ is one of $S_{1}, S_{2}, \ldots, S_{r}$, say $S=S_{1}$. By result of Gaschütz $[1](\operatorname{see}[4,6])$,

$$
d\left(B^{n}\right)=\max \left(d(B), d\left(S_{1}^{\lambda_{1} n}\right), \ldots, d\left(S_{r}^{\lambda_{r} n}\right)\right)
$$

for all $n$. Reasoning as in the first part of the proof, we conclude that there is a number $L$ depending only on $G$ such that

$$
d\left(B^{n}\right)=\max \left(d(B), d\left(S^{\lambda_{1} n}\right)\right)
$$

for all $n \geqq L$. Thus $d\left(B^{n}\right)=d\left(S^{\lambda_{1} n}\right)$ provided $n \geqq L$ and $d\left(S^{\lambda_{1} n}\right) \geqq d(G)$. Since this latter holds whenever $\log _{s}\left(\lambda_{1} n\right) \geqq d(G)$, and $\lambda_{1} \leqq \lambda$, the proof of Theorem $\mathrm{A}$ is complete.

THEOREM B. Let $B$ be a finite non-abelian simple group, $t$ an integer at least 1 , let $B_{1}, B_{2}, \ldots, B_{t}$ be groups isomorphic to $B$, and $G$ be their free product $B_{1} \star B_{2} \star \ldots \star B_{t}$. If $a=\mid$ Aut $B \mid$, there are precisely $\sum_{r=1}^{t}\left(\begin{array}{l}t \\ r\end{array}\right) a^{r-1}$ different normal subgroups $N$ of $G$ such that $G / N \cong B$.

Corollary. The highest power of $B$ that is homomorphic image of $G$ is $B^{\lambda}$, where $\lambda=\sum_{r=1}^{t}\left(\begin{array}{l}t \\ r\end{array}\right) a^{r-1}$.

Proof of Theorem B: Since each $B_{i}$ is simple, $N \cap B_{i}=1$ or $N \supseteq B_{i}$ for every normal subgroup $N$ of $G$. The proof proceeds by showing that for every $r$-element subset $K=\left\{i_{1}, i_{2}, \ldots, i_{r}\right\}$ of the set $I=\{1,2, \ldots, t\}$, there are precisely $a^{r-1}$ elements in the set

$$
U(K)=\left\{N \mid N \unlhd G \& G / N \cong B \&\left(k \in K \rightarrow N \cap B_{k}=1\right) \&\left(j \in I \backslash K \rightarrow N \supseteq B_{j}\right)\right\}
$$


Since there are $\left(\begin{array}{l}t \\ r\end{array}\right) r$-element subsets $K$ of $I$, summation over $r$ and $K$ will give the required result.

Two cases arise.

Case 1. $r=1$. A routine calculation shows that a non-trivial normal subgroup $N$ of $G$ is in $U(\{k\})$, for $k \in I$, if and only if it is the normal closure of $\bigcup_{j \neq k} B_{j}$. Thus $U(\{k\})$ has a single nember, as required.

Case 2. Suppose that $2 \leqq r \leqq t$ and that $K=\left\{k_{1}, k_{2}, \ldots, k_{r}\right\}$ is an $r$-element subset of $I$. Case 2 will follow when we establish a bijection between $U(K)$ and the set

$$
V=\left\{\left(\alpha_{2}, \cdots, \alpha_{r}\right) \mid \alpha_{i} \in \operatorname{Aut} B\right\}
$$

For each $N$ in $U(K)$ and each $i$ in $K$, the fact that $N \cap B_{i}$ is trivial implies that $N B_{i} / N$ is isomorphic to $B_{i}$ and thus to $G / N$. Thus, since $G / N$ is finite, $N B_{i}=G=$ $N B_{j}$ for all $i, j$ in $K$. So, for each $b \in B$ and each $j$ such that $2 \leqq j \leqq r$, there is a unique $b \alpha_{j}$ in $B$ for which

$$
N\left(b \psi_{i}\right)=N\left(\left(b \alpha_{j}\right) \psi_{j}\right)
$$

where $\psi_{i}$ is the isomorphism from $B$ to $B_{k_{i}}$. Because $N \cap B_{i}$ is trivial for each $i$ in $K$, it follows that the maps $\alpha_{j}: B \rightarrow B$ so defined are automorphisms of $B$; and $\theta$ defined by $N \theta=\left(\alpha_{2}, \ldots, \alpha_{r}\right)$ is a mapping from $U(K)$ to $V$.

Conversely, given $\left(\beta_{2}, \ldots, \beta_{r}\right) \in V$, let $T$ be the normal closure in $G$ of the set $\left\{\left(b \psi_{i}\right)^{-1}\left(b \beta_{j} \psi_{j}\right) \mid b \in B, 2 \leqq j \leqq r\right\}$, where the $\psi_{i}$ are as above. Let $F$ be the normal closure in $G$ of $\bigcup_{j \notin K} B_{j}$, and $M\left(\beta_{2}, \ldots, \beta_{r}\right)$ the product $T F$. Then $M\left(\beta_{2}, \ldots, \beta_{r}\right)$ is an element in $U(K)$ whose image under $\theta$ is $\left(\beta_{2}, \ldots, \beta_{r}\right)$. Moreover if $N$ is in $U(K)$ and $N \theta=\left(\alpha_{2}, \ldots, \alpha_{r}\right)$, then $M\left(\alpha_{2}, \ldots, \alpha_{r}\right)=N$, so that $\theta$ is bijective.

The Corollary follows since all the factor-groups $G / N$ are simple.

Proof of 'Theonem C: Let $G$ be a finitely generated perfect group with $m=$ $\mu(G)<\infty$, let $\left\{1, x_{2}, \ldots, x_{m}\right\}$ be a Meier set for $G$, and let $W=\left\{\left(g_{i 1}, g_{i 2}, \ldots, g_{i n}\right) \mid\right.$ $\left.1 \leqq i \leqq d\left(G^{n}\right)=d\right\}$ be a minimal generating set for $G^{n}$. The proof is very similar to that of Theorem 4.2 in [8].

We prove by induction on $r$ that the subgroup $H$ of $G^{n r}$ generated by the following elements is $G^{n r}$ :

$$
\begin{aligned}
a_{i} & =\left(g_{i 1}, g_{i 2}, \ldots, g_{i n}, g_{i 1}, \ldots, g_{i n}, \ldots, g_{i 1}, \ldots, g_{i n}\right), \quad 1 \leqq i \leqq d \\
a_{d+1} & =\left(1,1, \ldots, 1, x_{2}, \ldots, x_{2}, \ldots, x_{r}, \ldots, x_{r}\right),
\end{aligned}
$$

each with $r$ blocks of $n$ components. 
The result if trivial for $r=1$. Suppose that $r>1$ and that the result is established for smaller Meier sets. For arbitary $g_{1}, g_{2}, \ldots, g_{n}$ in $G, H$ contains

$$
\left(g_{1}, g_{2}, \ldots, g_{n}, g_{1}, \ldots, g_{n}, \ldots, g_{1}, \ldots, g_{n}\right)
$$

since $W$ generates $G^{n}$. In particular, $H$ contains

$$
h=\left(x_{2}, x_{2}, \ldots, x_{2}, x_{2}, \ldots, x_{2}, x_{2}, \ldots, x_{2}\right)
$$

and

$$
h a_{d+1}^{-1}=\left(x_{2}, x_{2}, \ldots, x_{2}, 1, \ldots, 1, x_{2} x_{3}^{-1}, \ldots, x_{2} x_{3}^{-1}, \ldots, x_{2} x_{r}^{-1}, \ldots, x_{2} x_{r}^{-1}\right) .
$$

But $\left\{1, x_{2} x_{3}^{-1}, \ldots, x_{2} x_{r}^{-1}\right\}$ is a Meier set for $G$ consisting of $r-1$ elements, and thus $\left\{a_{1}, a_{2}, \ldots, a_{d}, h a_{d+1}^{-1}\right\}$ generates $G^{n(r-1)}$ in the last $n(r-1)$ components. Thus for arbitary $g$ in $G$, and any $i$ such that $n+1 \leqq i \leqq n r, H$ contains an element of the form

$$
m_{i}=(\star, \star, \ldots, \star, 1, \ldots, 1, g, 1, \ldots, 1)
$$

where $g$ is in the $i$-th position and the stars are unknown. But

$$
\left[a_{d+1}, m_{i}\right]=\left(1, \ldots, 1,\left[x_{j_{i}}, g\right] 1, \ldots, 1\right)
$$

and $\left[x_{j_{i}}, G\right]=G$, so that $H$ contains $(1, \ldots, 1, g, 1, \ldots, 1)$, and so $H$ contains the whole of the $i$-th component. A glance at the form of $W$ shows that $H=G^{n r}$, and the theorem follows.

Proof of Theorem D: Here $G$ is a finite perfect group and $s$ is the smallest order of a simple image of $G$, and we wish to show that $\mu(G)=s$. Firstly, it is routine to show that epimorphisms carry Meier sets injectively into Meier sets, so certainly $\mu(G) \leqq s$; and we have to establish the existence of a Meier set of cardinal $s$.

This we du by induction on $|G|$. The result is clear for simple groups, so let $G$ be a non-simple group, $M$ a normal subgroup of $G$ so that $G / M$ is simple of order $s$, and suppose the result holds for groups of smaller order than $G$. Let $N$ be a normal subgroup of $G$ that is minimal among the non-trivial normal subgroups contained in $M$. Then $G / N$ is smaller than $G$ and $s$ is the smallest order of a simple image of $G / N$, so that by induction there is a Meier set $\left\{N, N x_{2}, \ldots, N x_{s}\right\}$ of $G / N$. Thus $G=N\left\langle x_{i}\right\rangle^{G}=N\left(x_{i} x_{j}^{-1}\right\rangle^{G}$ for different $i$ and $j$ between 1 and $s$. Two cases arise.

Case 1. If the $N \cap\left\langle x_{i}\right)^{G}$ and the $N \cap\left\langle x_{i} x_{j}^{-1}\right\rangle^{G}$ are all non-trivial, then by the minimality of $N,\left\langle x_{i}\right\rangle^{G} \geqq N$ and $\left\langle x_{i} x_{j}^{-1}\right\rangle^{G} \geqq N$ for all $i$ and $j$ so that $G=\left\langle x_{i}\right\rangle^{G}=$ $\left\langle x_{i} x_{j}^{-1}\right\rangle^{G}$ and $\left\{1, x_{2}, \ldots, x_{s}\right\}$ is a Meier set for $G$. 
Case 2. One of the above intersections is trivial, so that we can write $G=N \times T$. Both $N$ and $T$ are perfect, all their simple images are of order at most $s$, and $T$ has a simple image of order $s$, since $G / M=T M / M \cong T / T \cap M$. Thus by the inductive hypothesis $\mu(N) \geqq s$ and $\mu(T)=s$. If $\left\{1, h_{2}, \ldots, h_{s}, \ldots\right\}$ is a Meier set for $N$ and $\left\{1, g_{2}, \ldots, g_{s}\right\}$ is a Meier set for $T$, a very simple argument shows that $\left\{(1,1),\left(g_{2}, h_{2}\right), \ldots,\left(g_{s}, h_{s}\right)\right\}$ is a Meier set for $N \times T=G$. This completes Case 2 and the proof of the theorem.

Combining Theorems $\mathrm{C}$ and $\mathrm{D}$, we get that $d\left(G^{n}\right)<d(G)+\log _{s} n+1$ for a finite perfect group $G$ with $s$ the smallest order of a simple image of $G$. This is Lemma 2 of [5]; the proof given here is more elementary.

\section{REFERENCES}

[1] W. Gaschütz, 'Zu einem von B.H. und H. Neumann gestellten Problem', Math. Nachr. 14 (1955), 249-252.

[2] P. Hall, 'The Eulerian functions of a group', Quart. J. Math. (Oxford) 7 (1936), 134-151.

[3] J. Wiegold, 'Growth sequences of finile groups', J. Austral. Math. Soc. 17 (1974), 133-141.

[4] J. Wiegold, 'Growth sequences of finite groups II', J. Austral. Math. Soc. 20 (1975), 225-229.

[5] J. Wiegold, 'Growth sequences of finite groups III', J. Austral. Math. Soc. 25 (1978), 142-144.

[6] J. Wiegold, 'Growth sequences of finile groups IV', J. Austral. Math. Soc. 29 (1980), 14-16.

[7] D. Meier and James Wiegold, 'Growth sequences of finite groups V', J. Austral. Math. Sac. Ser. A. 31 (1981), 374-375.

[8] James Wiegold and J.S. Wilson, 'Growth sequences of finitely generated groups', Arch. Math. 30 (1978), 337-343.

Departinent of Mathematics

University of Zimbabwe

Harare

Zimbabwe
School of Mathematics

University of Wales

College of Cardiff

Senghenydd Road

Cardiff, CF2 4 AG

United Kingdom 